\title{
Alta incidencia del acto didáctico en la desmotivación por el aprendizaje del inglés, reconocida por profesores y estudiantes
}

Carlina Garavito de Archila'

carlina.garavito@doctorado.unini.edu.mx

carlinag.49@hotmail.com

https://orcid.org/0000-0001-5257-2674

Juliana Azevedo-Gomes ${ }^{\top}$

prof.juliana.azevedo@gmail.com

http://orcid.org/0000-0003-0192-5323

\section{https://doi.org/10.22209/rhs.v9n2a06}

Recibido: julio 22 de 2021

Aceptado: noviembre 2 de 2021

\section{Res u m e n}

La desmotivación por el aprendizaje del inglés en los estudiantes del nivel Media Vocacional $\left(10^{\circ} \mathrm{y}\right.$ $11^{\circ}$ ) en el Colegio Nuestra Señora de Fátima, de Onzaga, Santander, Colombia, nos llevó a realizar la presente investigación, cuyo objetivo fue evaluar la incidencia del acto didáctico en este problema y elaborar un plan de estrategias docentes, con base en la motivación por el aprendizaje del inglés, utilizando las nuevas tecnologías de información y comunicación, dirigido al nivel de educación Media Vocacional, para mejorar la calidad y con ella la motivación. Metodológicamente se recurrió a la

1 Universidad Internacional Iberoamericana (UNINI), México.
High incidence of the didactic act on demotivation in learning English as acknowledged by teachers and students investigación con enfoque mixto, y se ubicó dentro de la llamada investigación socio-educativa. Se utilizaron como técnicas la observación, el análisis documental, la entrevista (semi-estructurada y estructurada). Los instrumentos aplicados fueron el cuestionario, la encuesta, la escala de Likert y se tuvo en cuenta el tipo de muestreo probabilístico aleatorio simple. Los resultados muestran el cumplimiento de la hipótesis planteada con una alta incidencia del acto didáctico en la desmotivación por el aprendizaje del inglés, reconocida por profesores y estudiantes, concluyendo así la existencia de un acto didáctico frío humanamente, una comunicación inadecuada y la incorrecta interpretación de las demás características del acto didáctico.

Palabras clave: didáctica del inglés; tecnologías de la información y la comunicación; aprendizaje significativo; acto didáctico; desmotivación/ motivación.

\section{Abstrac}

Demotivation in learning English among vocational school students (grades 10 and 11) at Nuestra Señora de Fátima High School (Onzaga, Santander, Colombia) led us to undertake this research study. Its goal was to assess the incidence of the didactic act on this problem as well as to develop a plan of 
teaching strategies aimed at fostering motivation to learn English, using new ICT tools. The plan intended to improve the education quality and motivation at the vocational high school level. A mixed methodology was used in this research study, which was based on a socio-educational model. The techniques used included observation, document analysis and interviews (both semistructured and structured). The instruments administered were a questionnaire, a survey and a Likert scale. The selection of the samples involved the use of simple probability random sampling. The results confirmed the proposed hypothesis that the didactic act has a high incidence on demotivation in learning English as acknowledged by teachers and students. The conclusion highlights the existence of a cold didactic relation, poor communication and misinterpretation of the remaining characteristics of the didactic act.

Keywords: didactics of English; information and communication technologies (ICT); significant learning; didactic action; demotivation / motivation.

\section{Introducción}

L a apatía, desinterés o desmotivación por el Japrendizaje del inglés, reflejado en la actitud del estudiante, que se puede ver en diferentes documentos tanto de la institución, como en reportes de las pruebas Saber 11, sumado a que en la historia del Colegio no hay más de un egresado que haya seguido una licenciatura en idiomas motivan esta investigación, que es de alta relevancia académica. Para el aprendizaje se requieren personas motivadas con actitudes positivas o predisposiciones y formas habituales de sentir, pensar y actuar, tanto del que enseña como del que aprende, de lo contrario, siempre se crearán problemas en todos los aspectos y se limitará el progreso y desarrollo. Aprender inglés hoy es más importante que nunca, debido a que la globalización lo exige en todo contexto.

Los resultados de algunas investigaciones científicas demuestran que "estudiar inglés produce ciertos estímulos en el cerebro que nos ayudan a mejorar nuestras habilidades en otras áreas como son la creativa, la resolución de problemas, el razonamiento o la habilidad mental" (Vargas, 2017. párr. 9). "Aprender nuevos idiomas mejora la reserva cognitiva y la plasticidad cerebral, las habilidades verbales, la velocidad de procesamiento mental y la flexibilidad cognitiva, lo cual repercute en una mayor eficiencia de las funciones ejecutivas y capacidad de análisis" (Vega, 2020). Otros como Smart Academia, (2021); Chaparro, (2018); Closer English, (2019); también coinciden con las ventajas, los beneficios y resultados de tener un cerebro bilingüe, lo que nos lleva a la conclusión que estudiar y aprender inglés mejora, protege, potencia y desarrolla nuestro cerebro. Por tanto, resulta indispensable hoy en día para un avance profesional, además de las ventajas que ofrece en materia de salud.

Existen muchos estudios sobre la enseñanza del inglés, sin embargo, no tocan el acto didáctico, utilizando un enfoque científico; de ahí la pregunta problema: ¿de qué manera el acto didáctico es la causa de la desmotivación por el aprendizaje del inglés en los estudiantes del nivel Media Vocacional del Colegio Nuestra Señora de Fátima? Se puso en consideración la siguiente hipótesis: "la desmotivación hacia el aprendizaje del inglés depende del acto didáctico". El objetivo principal fue evaluar la incidencia del acto didáctico en la 
desmotivación por el aprendizaje del inglés en el nivel de educación Media Vocacional, para mejorar la calidad y con ella la motivación.

\section{Materiales y métodos}

"Investigamos para descubrir, para responder a interrogantes que nos planteamos ante hechos sin explicación aparente, ante discrepancias entre teoría y práctica o ante lagunas del conocimiento limitado" (Busot, 1985, p. 12). El propósito del diseño de esta investigación fue elegir los métodos y técnicas y combinarlos de manera razonablemente lógica, para manejar el problema de manera eficiente. Técnicas utilizadas: la observación, el análisis de documentos, la entrevista y la sensibilización. Instrumentos aplicados: el cuestionario, la encuesta de tipo mixto, la escala de Likert, y la sensibilización. Metodológicamente se empleó la investigación cualitativa, con enfoque mixto, cuya meta fue utilizar las fortalezas de ambos tipos de indagación, combinándolos y tratando de minimizar sus debilidades potenciales (Hernández Sampieri, 2010, p. 544), y de triangulación, teniendo en cuenta la neutralidad, fiabilidad, validez y generalización. Según el grado de manipulación de las variables, la investigación fue cuasi-experimental y, según el tipo de inferencia, la investigación fue de tipo deductivo. El tipo de estudio fue el de campo pasando por: explorativo, descriptivo, y correlacional. La naturaleza de la investigación fue con enfoque cualitativo y se ubicó dentro la llamada investigación socio-educativa. La población de estudio fue de 52 estudiantes, del nivel Media Vocacional y 20 profesores. Para obtener las muestras se seleccionó el tipo de muestreo probabilístico aleatorio simple, obteniendo las muestras de 22 estudiantes y 13 docentes.
El principal objetivo fue evaluar la incidencia del acto didáctico en la desmotivación por el estudio y aprendizaje del inglés y, así mismo, poner a prueba la hipótesis: "La desmotivación por el aprendizaje del inglés depende del acto didáctico respectivo".

\section{Población y muestra}

Se trabajó con dos grupos: la población de estudio (1) fueron 52 estudiantes del nivel Media Vocacional, designada por decisión razonada en función de sus características y con madurez para un mejor diagnóstico. La población de estudio (2) fueron los 20 profesores que laboraban en el Colegio Nuestra Señora de Fátima, quienes estuvieron muy relacionados con el estudio y recibieron jornadas de sensibilización y capacitación sobre las variables: acto didáctico y desmotivación por el aprendizaje del inglés.

Para obtener las muestras se aplicó el tipo de muestreo probabilístico aleatorio simple y para determinar el tamaño de la muestra de la población tanto de estudiantes como de profesores, se utilizó la siguiente fórmula, resultando 22 estudiantes y 13 docentes como vemos en la Ecuación 1:

\section{Ecuación 1}

Muestreo aleatorio simple.

$$
\mathrm{n}=\frac{Z^{2} \times p \times q \times N}{N \times E^{2}+Z^{2} \times p \times q}
$$

Nota. Fuente: Otzen, T. \& Manterola C. (2017). Técnicas de muestreo sobre una población a estudio. 
Los parámetros fueron los siguientes:

$\mathrm{n}=$ Muestra

$p=$ Probabilidad a favor $=0,5$ (predeterminada)

$q=$ Probabilidad en contra $=(1-p)=0,5$

$Z=$ Nivel de confianza $=1,96$ (predeterminada)

$\mathrm{E}=$ Margen de Error $5 \%=0,05$ (predeterminada)

$\mathrm{N}=$ Población finita $=52\left(1^{\circ}\right.$ población tomada para el estudio)

$\mathrm{N}=$ Población finita $=20\left(2^{\circ}\right.$ población tomada para el estudio)

\section{Variables}

Las variables que intervinieron en el estudio fueron: el acto didáctico, como variable independiente y la desmotivación hacia el estudio del inglés como variable dependiente. Se eligieron las siguientes definiciones conceptuales:

Se entiende el acto didáctico como

el momento sublime de la labor docente, el encuentro personal entre educador y educando mediatizado por la enseñanza y el aprendizaje de comportamientos, actitudes, contenidos, habilidades y saberes. Debe estar revestido de atributos de calidad que lo hagan digno, formador, motivante y casi sagrado. (La Nación, 2017)

Otra definición tenida en cuenta: "sistema de comunicación intencional que se produce en un marco institucional y en el que se generan estrategias encaminadas a provocar el aprendizaje" (Contreras, 1994, p. 23). El Marco teórico del acto didáctico lo constituyen los elementos fundamentales: docentes, discentes, los objetivos de aprendizaje, la materia, los métodos de enseñanza y el contexto de aprendizaje; y los elementos complementarios: qué deben aprender, cómo deben aprender, para qué deben aprender, cómo evaluar los logros. Los momentos didácticos son la planificación, implementación y evaluación.

La desmotivación, según la RAE (2017), es "la falta o pérdida de la motivación". Ahora bien: ¿qué es la motivación? Maslow (1991, p. 264) la define a lo largo de sus investigaciones como el impulso del ser humano para satisfacer sus necesidades dividiendo las mismas en necesidades básicas - relacionadas con la supervivencia y seguridad-y superiores -referentes con la autorrealización personal y social- (Esparcia, 2018).

\section{Técnicas e instrumentos de investigación}

Las técnicas utilizadas fueron la observación y la entrevista, estructuradas y no estructuradas, a través del cuestionario y la encuesta, el análisis documental y análisis de contenidos.

\section{Validación de documentos}

Para validar los instrumentos se siguió el proceso indicado en Hernández Sampieri (2017). Además, las magísteres Magdalena Gómez León y Eneida Deyanire Ramos Lukerna, docentes Licenciadas en Lenguas, dieron su aprobación para aplicar los cuestionarios o encuestas, luego de ser probados. Haciendo el recorrido anterior se estableció una síntesis dialéctica, pues el objetivo no era solo describir el fenómeno educativo, sino, además, aportar mecanismos de sensibilización para facilitar que el grupo de profesores reaccionen frente a esa inercia e indiferencia por los resultados con la que viven año tras año. 
También se quería formular un plan de estrategias docentes, con base en la motivación por el aprendizaje del inglés, utilizando las nuevas tecnologías de la información para el aprendizaje significativo de este idioma en el desarrollo del acto didáctico.

\section{Procedimiento del desarrollo de los objetivos específicos}

Objetivo nro. 1. Conocer los niveles de desmotivación hacia el estudio del inglés en los estudiantes del Colegio Nuestra Señora de Fátima.

Tipo de estudio: exploratorio. Modalidad de la investigación: documental y de campo.

Método: análisis sintético. Herramientas de investigación: revisión bibliográfica de textos, documentos de la Web, planes de estudio de la institución, observación directa acerca de evidencias (actividades, trabajos, horarios, uso de recursos, ambientes, relaciones profesor alumno, etc.), relacionados con la materia de inglés; además, se utilizó la entrevista en forma de cuestionario estructurado, para conocer los niveles de desmotivación.

Objetivo nro. 2. Identificar los niveles de calidad del acto didáctico, encuesta aplicada a los profesores.

Tipo de estudio: exploratorio sobre los niveles de calidad del acto didáctico (tomado como el dominio y uso adecuado de los elementos, complementos y momentos del acto didáctico, permitiendo determinar los problemas fundamentales que inciden en la desmotivación del estudiantado. Modalidad de la investigación: documental y de campo; también se necesitó de la encuesta y la entrevista para que el investigador pudiera forjar una opinión del funcionamiento de la pedagogía y la didáctica, en el desarrollo del acto didáctico en la enseñanza del inglés. Método: analítico sintético. Herramientas de investigación: la entrevista a través de la encuesta.

Objetivo nro. 3. Determinar la relación que existe entre los niveles de desmotivación de los estudiantes y los niveles de calidad del acto didáctico. Encuesta aplicada a los estudiantes.

Tipo de estudio: el correlacional. (medir las dos variables). Modalidad de la investigación: documental y de campo, Método: sintético y correlacional (para observar las causas, la naturaleza y los efectos). Herramientas de la investigación: la entrevista a través de la encuesta estructurada (escalamiento tipo Likert), el cual sirve para medir las actitudes de los profesores en el desarrollo del acto didáctico.

Objetivo nro. 4. Retroalimentar la importancia de los elementos, complementos y momentos del acto didáctico, a través de la sensibilización.

Tipo de estudio: exploratorio. Investigación educativa explicativa. Sabino nos dice: "son aquellos trabajos, donde nuestra preocupación se centra en determinar los orígenes o causas de un determinado conjunto de fenómenos" (1996, p. 106). Modalidad de la investigación: investigación orientada a la formación del recurso humano, ya que se detectó que no hay unidad de criterios sobre el concepto del acto didáctico. Método: empírico analítico (además, con la finalidad de establecer los mecanismos para validar la hipótesis). Herramientas de la investigación: la sensibilización a través de una exposición y, al finalizar, se realizó una evaluación del antes y el después sobre el tema: Retroalimentar la importancia del 
dominio del acto didáctico de manera integral.

Objetivo nro. 5. Actualizar y profundizar en los conceptos clave que integran el acto didáctico de calidad. Este objetivo se desarrolló con las mismas características del objetivo anterior, ya que la metodología era la misma.

Una vez terminada la sensibilización, se aplicó una pequeña encuesta con el objetivo de evaluar el cumplimiento y sus efectos del objetivo nro.5 y validar una vez más la hipótesis planteada. "La desmotivación hacia el estudio o aprendizaje del inglés depende del acto didáctico".

Objetivo nro. 6. Construir un plan de estrategias que mejoren la didáctica en la enseñanza del inglés para mejorar la motivación por su aprendizaje.

Tipo de estudio: descriptivo. Se describe el plan de estrategias docentes, con base en la motivación por el aprendizaje del inglés para ser desarrollada por los profesores en el ejercicio del acto didáctico con los estudiantes del nivel Media Vocacional y en los demás niveles. Modalidad de la investigación: estudio documental. Método: descriptivo. Herramientas de la investigación: contenidos relacionados con el objetivo de cada plan o unidad, computador, internet y conocimientos del investigador, obteniendo el siguiente plan:

Unidad 1: estrategias docentes para conocer a los estudiantes. Objetivo: conectar con los alumnos para conocer sus fortalezas y debilidades.

Unidad 2: estrategias docentes para mantener el entusiasmo y dedicar tiempo a cada estudiante. Objetivo: mantener el entusiasmo y gusto por la materia y por el legítimo placer de enseñar y dedicar tiempo a cada uno.
Unidad 3: estrategias docentes para mantener altas expectativas y para señalar la importancia de la asignatura. Objetivo: mostrar confianza y dar consejos prácticos a los estudiantes, para influir con altas expectativas y señalar la importancia de la asignatura del inglés.

Unidad 4: estrategias docentes para mantener la confianza, la seguridad, la autoestima, el respeto y sobre todo la motivación del estudiante por el estudio. Objetivo: propiciar confianza, seguridad, respeto, afecto y comprensión para mejorar la autoestima y motivación por el estudio.

Cada plan o unidad es para cuatro sesiones y contiene: contenidos, objetivos específicos, estrategias metodológicas, estrategias de evaluación y duración.

Análisis estadístico. Se hizo un sondeo bastante importante, tanto a estudiantes, como a profesores, para obtener conocimientos de ciertas realidades subjetivas como sentimientos y motivaciones; luego se dio paso a profundizar un poco más en la observación y en la entrevista a través del cuestionario y la encuesta con preguntas abiertas y cerradas. Además, se aplicó la escala de Likert, para recabar sobre las dos variables del estudio. Se terminó con un resumen, el cual se logró promediando los indicadores de cada componente del acto didáctico. También se desarrollaron las técnicas Focus group y Delphi, a través de la sensibilización, buscando recabar las opiniones en un ambiente relajado y las contrastantes en un punto de consenso. Se recogió la información, se sistematizó y algunas respuestas se graficaron sobre todo aquellas que daban directamente respuestas a cada uno de los objetivos. Por último, se elaboró un "Plan de estrategias docentes para mejorar la 
motivación por el aprendizaje del inglés en los estudiantes de Media Vocacional $\left(10^{\circ}\right.$ y $11^{\circ}$ ) del Colegio Nuestra Señora de Fátima", para ser ejecutado por los profesores en el ejercicio diario del acto didáctico.

\section{Resultados}

El hallazgo principal fue encontrar la "alta" incidencia del acto didáctico en la desmotivación de los estudiantes por el estudio del inglés, ya que los resultados clave de los objetivos específicos trazados fueron: los niveles de desmotivación en los estudiantes hacia el estudio del inglés estuvieron en "alto"; el nivel de calidad en que los profesores desarrollan el acto didáctico fue "regular"; la relación que existe entre los niveles de desmotivación de los estudiantes y los niveles de calidad del acto didáctico fue inversa y negativa; las sensibilizaciones sobre el acto didáctico tuvieron como resultado en un $100 \%$ que los docentes reconocieran que los aportes fueron muy importantes para mejorar la calidad del acto didáctico del inglés y de todas las áreas y que confirmaran que la desmotivación de los estudiantes por el estudio del inglés dependiera de acto didáctico. Además, los docentes hicieron observaciones y sugerencias muy importantes, con intención de seguir mejorando como: "muy importante este tipo de capacitaciones, nos muestra la realidad de nuestras aulas". "Recibir estas mismas orientaciones en las jornadas pedagógicas aplicables a la institución".
“Capacitaciones frecuentes, como esta”. "Hacer conversatorios frecuentes sobre estos temas". "Todo estuvo muy claro y coherente. Muchas gracias" (Profesores Colegio Nuestra Señora de Fátima, 2019). Se logró elaborar un plan de estrategias docentes, basadas en la motivación: "Plan de estrategias docentes para mejorar la motivación por el aprendizaje del inglés en los estudiantes de Media Vocacional $\left(10^{\circ}\right.$ y $\left.11^{\circ}\right)$ del Colegio Nuestra Señora de Fátima", para ser ejecutada por los profesores en el ejercicio diario del acto didáctico e ir mejorando la motivación del estudiante y la del mismo profesor.

Las siguientes tablas y gráficos, nos ilustran los anteriores hallazgos y otros que nos amplían y complementan, en cada uno de los objetivos de la investigación:

Primer objetivo. Conocer los niveles de desmotivación hacia el estudio del inglés en los estudiantes del Colegio Nuestra Señora de Fátima. (Encuesta aplicada a los estudiantes). (Ver tablas 1, 2, 3 y figuras 1, 2, 3).

Tabla 1. Estado de la motivación hacia el estudio del inglés

\begin{tabular}{|l|c|c|c|c|}
\hline $\begin{array}{c}\text { Niveles/ } \\
\text { respuesta }\end{array}$ & Buena & Regular & Mala & $\begin{array}{c}\text { Muy } \\
\text { mala }\end{array}$ \\
\hline Cantidad & 7 & 9 & 4 & 2 \\
\hline Porcentaje & $32 \%$ & $41 \%$ & $18 \%$ & $9 \%$ \\
\hline
\end{tabular}


Figura 1. Estados de la motivación

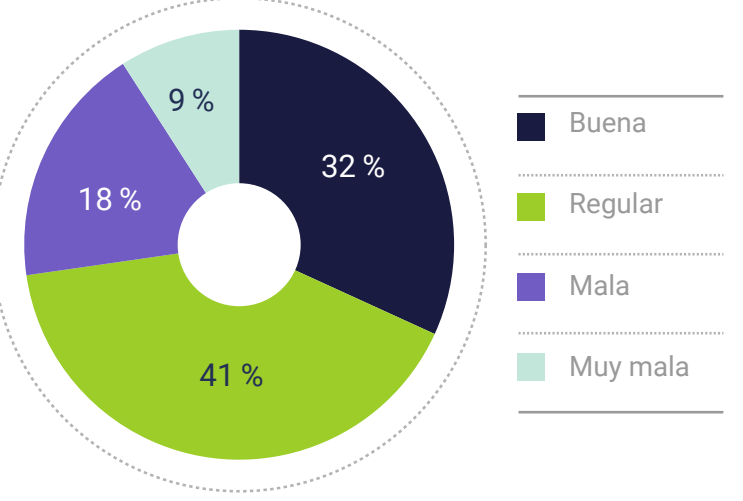

Tabla 2. La desmotivación depende del acto didáctico

\begin{tabular}{|l|c|c|c|}
\hline \multicolumn{1}{|c|}{ Respuestas } & Si & No & $\begin{array}{c}\text { No } \\
\text { contestan }\end{array}$ \\
\hline Cantidad & 13 & 7 & 2 \\
\hline Porcentaje & $59 \%$ & $32 \%$ & $9 \%$ \\
\hline
\end{tabular}

Figura 2. La desmotivación depende del acto didáctico

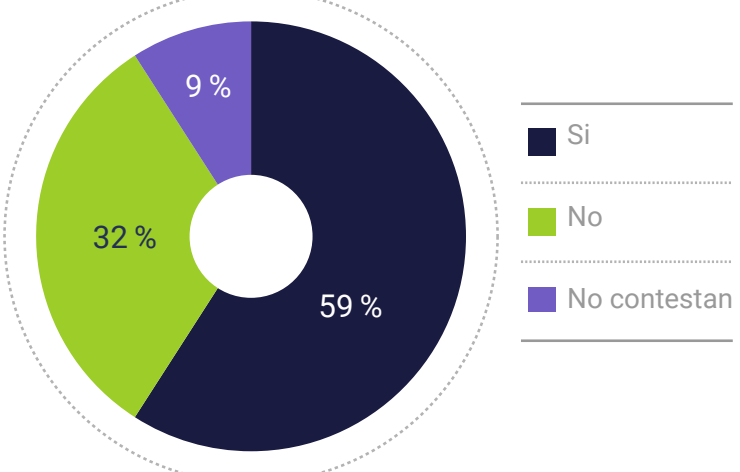

Tabla 3. Nivel de desmotivación frente al estudio o aprendizaje del inglés

\begin{tabular}{|l|c|c|c|c|c|}
\hline $\begin{array}{c}\text { Niveles/ } \\
\text { Alternativas }\end{array}$ & $\begin{array}{c}\text { Muy } \\
\text { Alto }\end{array}$ & Alto & Medio & Bajo & $\begin{array}{c}\text { Muy } \\
\text { Bajo }\end{array}$ \\
\hline Cantidad/F.A. & 1 & 13 & 4 & 2 & 2 \\
\hline Porcentaje & $5 \%$ & $59 \%$ & $18 \%$ & $9 \%$ & $9 \%$ \\
\hline
\end{tabular}

Figura 3. Niveles de desmotivación

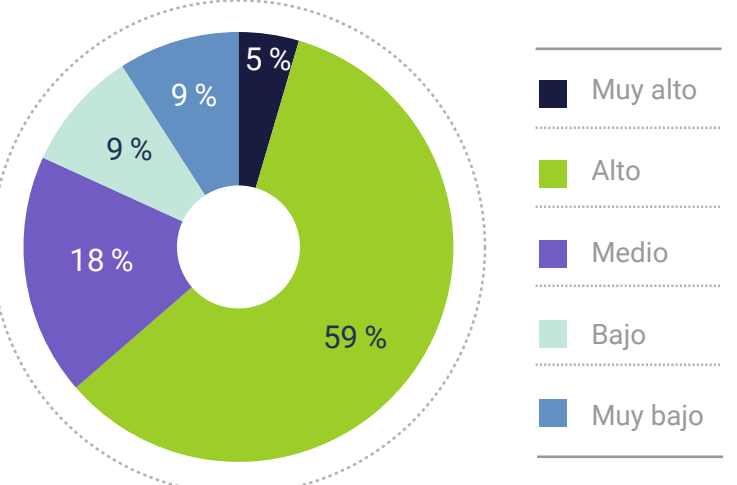

Segundo objetivo. Identificar los niveles de calidad del acto didáctico. (Encuesta aplicada a los profesores). (Ver tablas 4, 5, 6, 7 y figuras $4,5,6,7)$.

Tabla 4. Rendimiento académico de los estudiantes, sobre todo en inglés

\begin{tabular}{|l|c|c|c|}
\hline \multicolumn{1}{|c|}{ Respuestas } & Alto & Bajo & Insuficiente \\
\hline Cantidad & 4 & 7 & 2 \\
\hline Porcentaje & $31 \%$ & $54 \%$ & $15 \%$ \\
\hline
\end{tabular}

Figura 4. Rendimiento académico en inglés

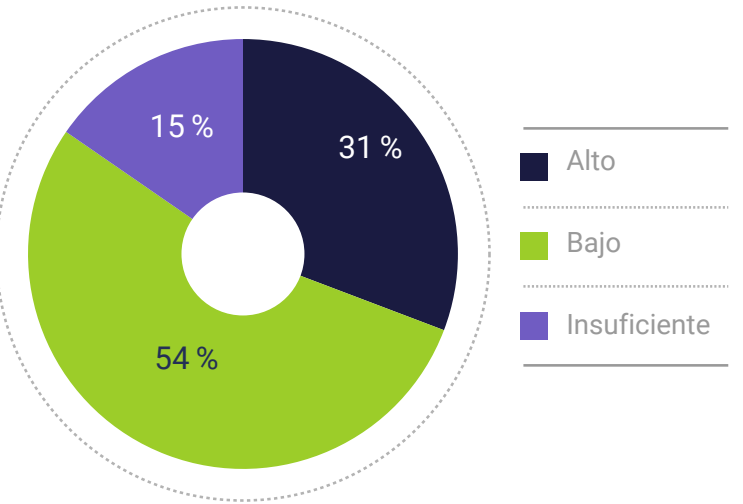


Tabla 5. Nivel de calidad en que desarrollan el acto didáctico

\begin{tabular}{|l|c|c|c|c|}
\hline \multicolumn{1}{|c|}{$\begin{array}{c}\text { Nivel de } \\
\text { calidad }\end{array}$} & Excelente & Bueno & Regular & Malo \\
\hline Cantidad (f.A.) & 1 & 4 & 6 & 2 \\
\hline Porcentaje & $8 \%$ & $31 \%$ & $46 \%$ & $15 \%$ \\
\hline
\end{tabular}

Figura 5. Nivel de calidad del acto didáctico

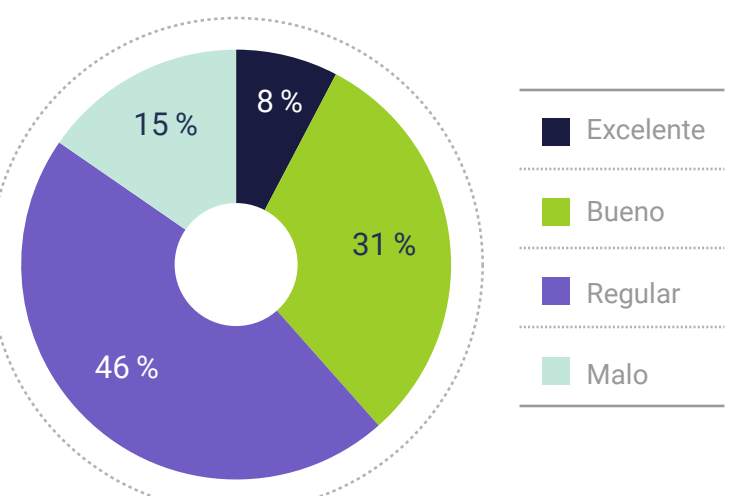

Tabla 6. La desmotivación hacia el estudio del inglés depende del acto didáctico

\begin{tabular}{|l|c|c|l|}
\hline Respuestas & Si & No & \multicolumn{1}{c|}{$\begin{array}{c}\text { Observacion de } \\
\text { un docente. }\end{array}$} \\
\hline Cantidad & 11 & 2 & $\begin{array}{l}\text { El acto didáctico } \\
\text { contiene los elementos } \\
\text { necesarios para el } \\
\text { aprendizaje de esta } \\
\text { lengua. La principal de } \\
\text { ellos: la motivación. }\end{array}$ \\
\hline Porcentaje & $\mathbf{8 5 \%}$ & $\mathbf{1 5 \%}$ & \\
\hline
\end{tabular}

Figura 6. La desmotivación depende del acto didáctico.

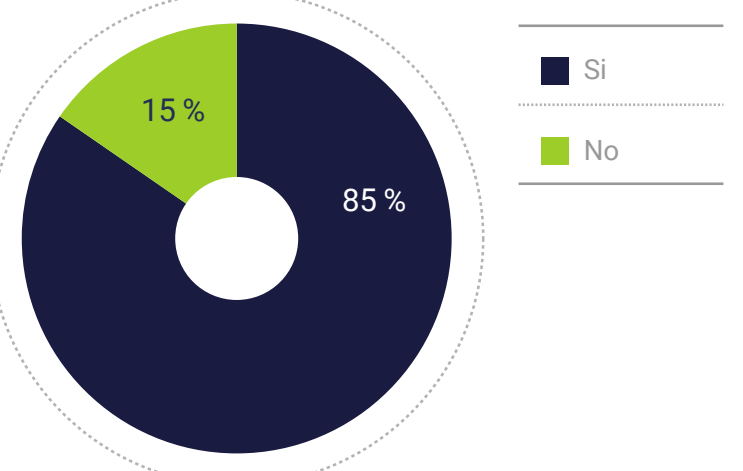

Tabla 7. Necesidad de una retroalimentación sobre el acto didáctico

\begin{tabular}{|l|c|c|}
\hline \multicolumn{1}{|c|}{ Respuestas } & Si & No \\
\hline Cantidad & 13 & 0 \\
\hline Porcentaje & $100 \%$ & $0 \%$ \\
\hline
\end{tabular}

Figura 7. Necesidad de retroalimentar el acto didáctico.

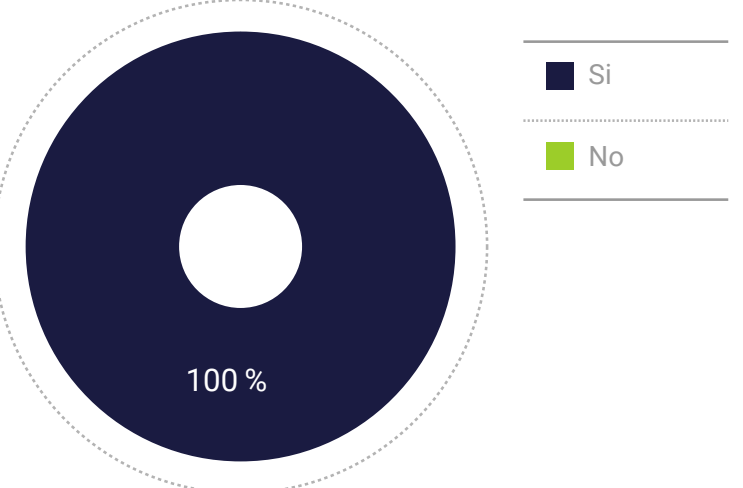

Tercer objetivo. Determinar la relación que existe entre los niveles de desmotivación de los estudiantes y los niveles de calidad del acto didáctico. (Encuesta escala de Likert aplicada a los estudiantes). (Ver tablas 8, y 9 y figura 8).

Tabla 8. Interpretación resumen de la encuesta de la escala de Likert

\begin{tabular}{|l|l|}
\hline \multicolumn{1}{|c|}{ Rango 1 (escala 1-3) } & \multicolumn{1}{|c|}{ Rango 2 (escala 4-5) } \\
\hline Alternativas: Nunca, A & Alternativas: Casi siempre, \\
\hline veces, Con frecuencia. & Siempre. \\
\hline $\begin{array}{l}\text { Categorías: Muy baja, } \\
\text { Baja, Media. }\end{array}$ & Categorías: Alta, Muy Alta. \\
\hline
\end{tabular}


Tabla 9. Resumen de la valoración de los componentes según promedio de sus indicadores en la encuesta

\begin{tabular}{|l|c|c|}
\hline \multicolumn{1}{|c|}{ COMPONENTES } & Rango 1 & $\begin{array}{c}\text { Rango 2 } \\
\text { Alta, Muy Alta. }\end{array}$ \\
\hline El Educador & Muy baja, Baja, Media & $39,22 \%$ \\
\hline El Discente & $61,11 \%$ & $77,5 \%$ \\
\hline Objetivos de Aprendizaje & $22,5 \%$ & 63,75 \\
\hline El Contexto & 36,25 & $63 \%$ \\
\hline Recursos Didácticos & $37 \%$ & $42 \%$ \\
\hline Estrategia Didáctica & $58 \%$ & $50 \%$ \\
\hline Clima de Aula & $50 \%$ & $41 \%$ \\
\hline Los Contenidos & $59 \%$ & $55 \%$ \\
\hline Relación Profesor-Alumno & $45 \%$ & $45,33 \%$ \\
\hline El Reconocimiento & $54,66 \%$ & $46 \%$ \\
\hline Contexto Familiar y del entorno & $54 \%$ & $36 \%$ \\
\hline
\end{tabular}

Figura 8. Resumen de los promedios de cada componente del acto didáctico.

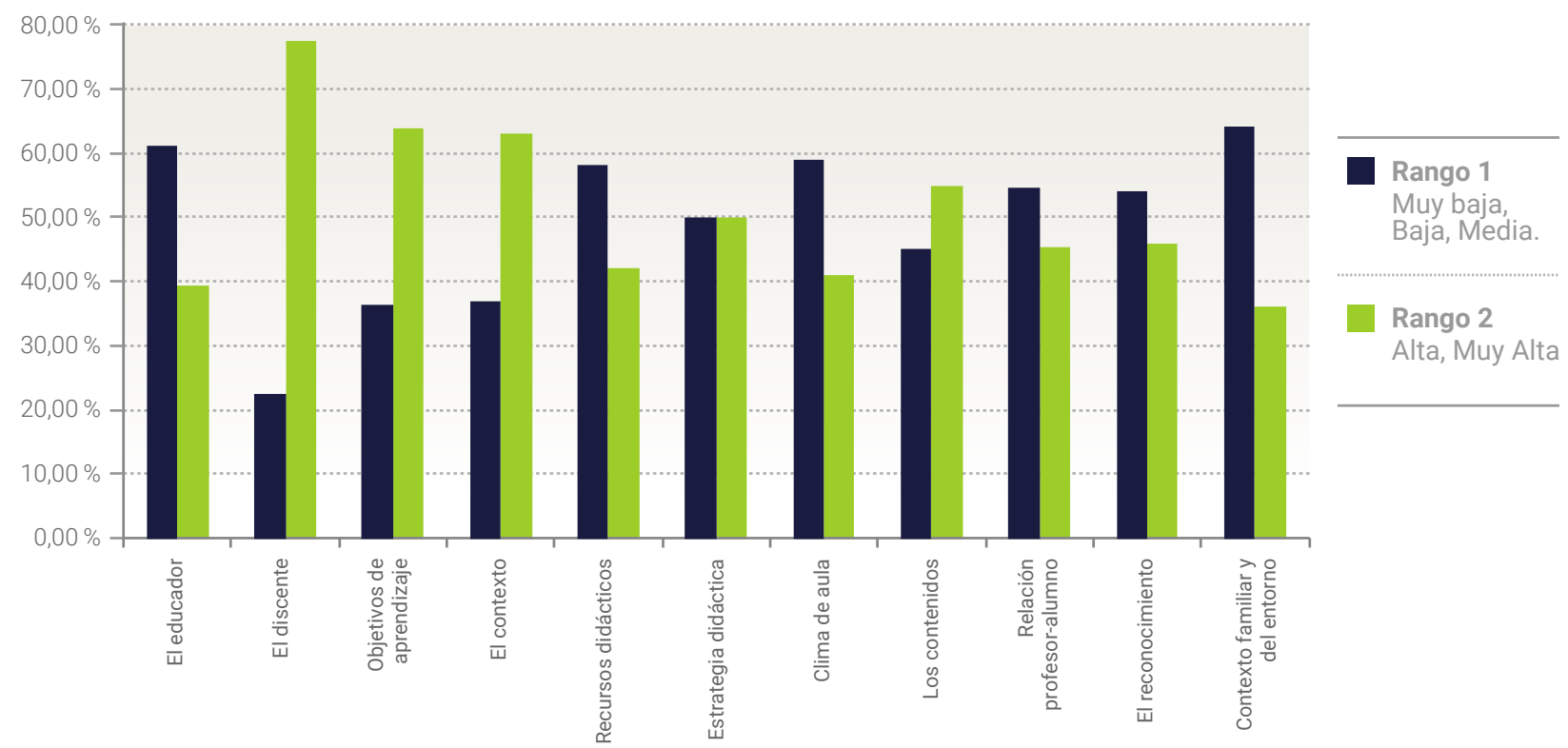

El acto didáctico para el estudio tiene $11 \mathrm{com}-$ ponentes, de los cuales 6 de ellos (color azul) están ubicados en el rango 1, lo que quiere decir que están en las categorías de calidad: (muy baja, baja y media) y son los componentes que más tienen que ver con la motivación o desmotivación del estudiante, como: el educador, recursos didácticos, estrategia didáctica, clima de aula, relación profesor alumno, reconocimiento y contexto familiar y del entorno. Podemos decir que existe una relación inversa y negativa.

Cuarto objetivo. Retroalimentar la importancia de los elementos, complementos y momentos del acto didáctico en los profesores. (Ver tablas 10,11 , y 12 y figuras 9,10 y 11 ). 
Tabla 10. Importancia del tema

\begin{tabular}{|l|c|c|c|c|}
\hline Alternativa & Claro & Oportuno & Importante & $\begin{array}{c}\text { Muy } \\
\text { importante }\end{array}$ \\
\hline Cantidad & 3 & 3 & 2 & 5 \\
\hline Porcentaje & $23 \%$ & $23 \%$ & $15 \%$ & $39 \%$ \\
\hline
\end{tabular}

Figura 9. Importancia del tema.

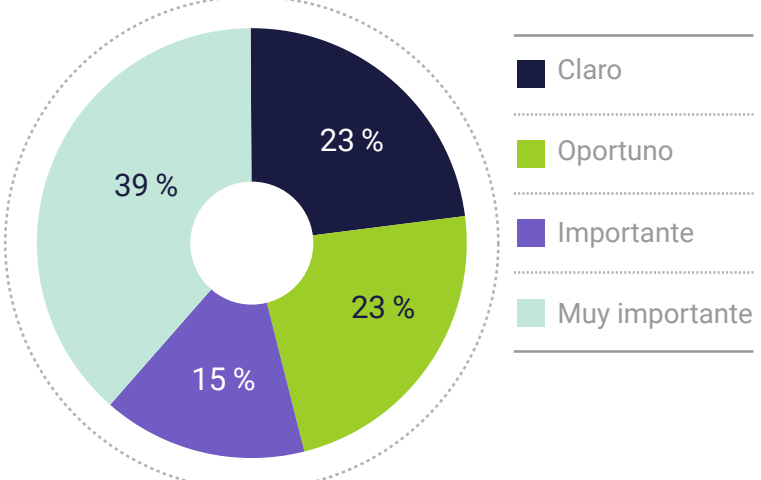

Tabla 11. La desmotivación por el estudio del inglés depende del acto didáctico

\begin{tabular}{|l|c|c|}
\hline \multicolumn{1}{|c|}{ Alternativas } & Sí & No \\
\hline Cantidad & 13 & 0 \\
\hline Porcentaje & $100 \%$ & $0 \%$ \\
\hline
\end{tabular}

Figura 10. La desmotivación depende del acto didáctico.

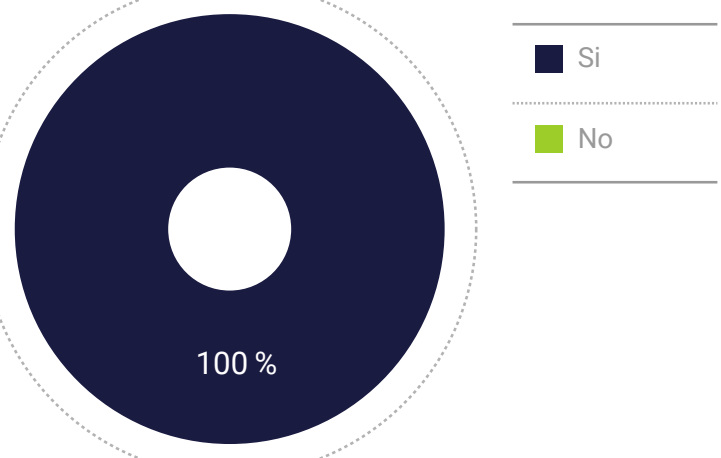

Tabla 12. Necesidad de redefinir los conceptos clave de los componentes del acto didáctico

\begin{tabular}{|l|c|c|}
\hline \multicolumn{1}{|c|}{ Alternativa/respuesta } & Si & No \\
\hline Cantidad & 13 & 0 \\
\hline Porcentaje & $100 \%$ & $0 \%$ \\
\hline
\end{tabular}

Figura 11. Necesidad de redefinir los conceptos clave de los componentes del acto didáctico.

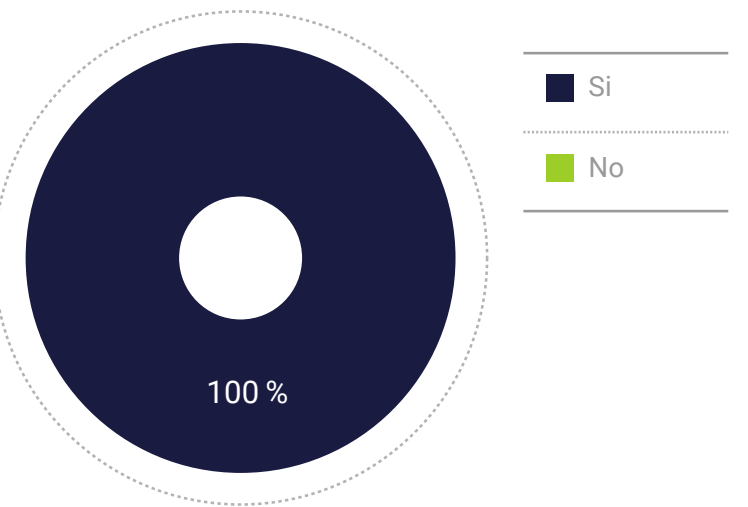

Quinto objetivo. Profundizar en los conceptos clave que integran el acto didáctico, con los profesores. (Ver tablas 13, 14, y 15 y figuras 12 y 13).

Tabla 13. Los aportes fueron importantes

\begin{tabular}{|l|c|c|}
\hline \multicolumn{1}{|c|}{ Alternativa/ respuesta } & Si & No \\
\hline Cantidad & 13 & 0 \\
\hline Porcentaje & $100 \%$ & $0 \%$ \\
\hline
\end{tabular}

Figura 12. Importancia de los aportes para mejorar la calidad del proceso enseñanza-aprendizaje.

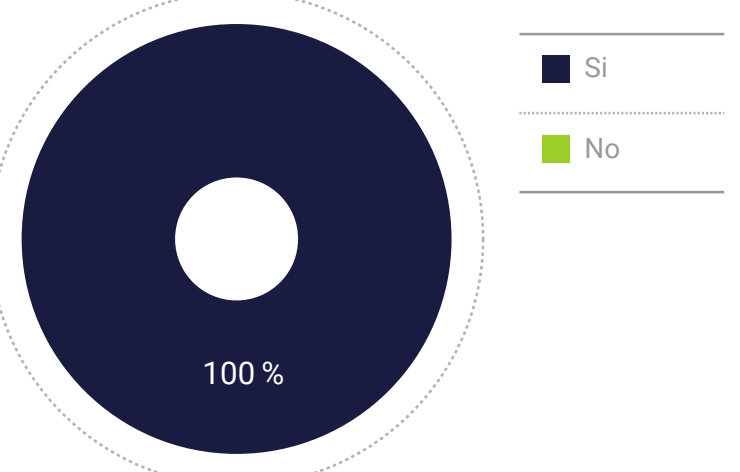

Además, los docentes registraron las siguientes observaciones: 
Figura 13. Observaciones de los docentes.

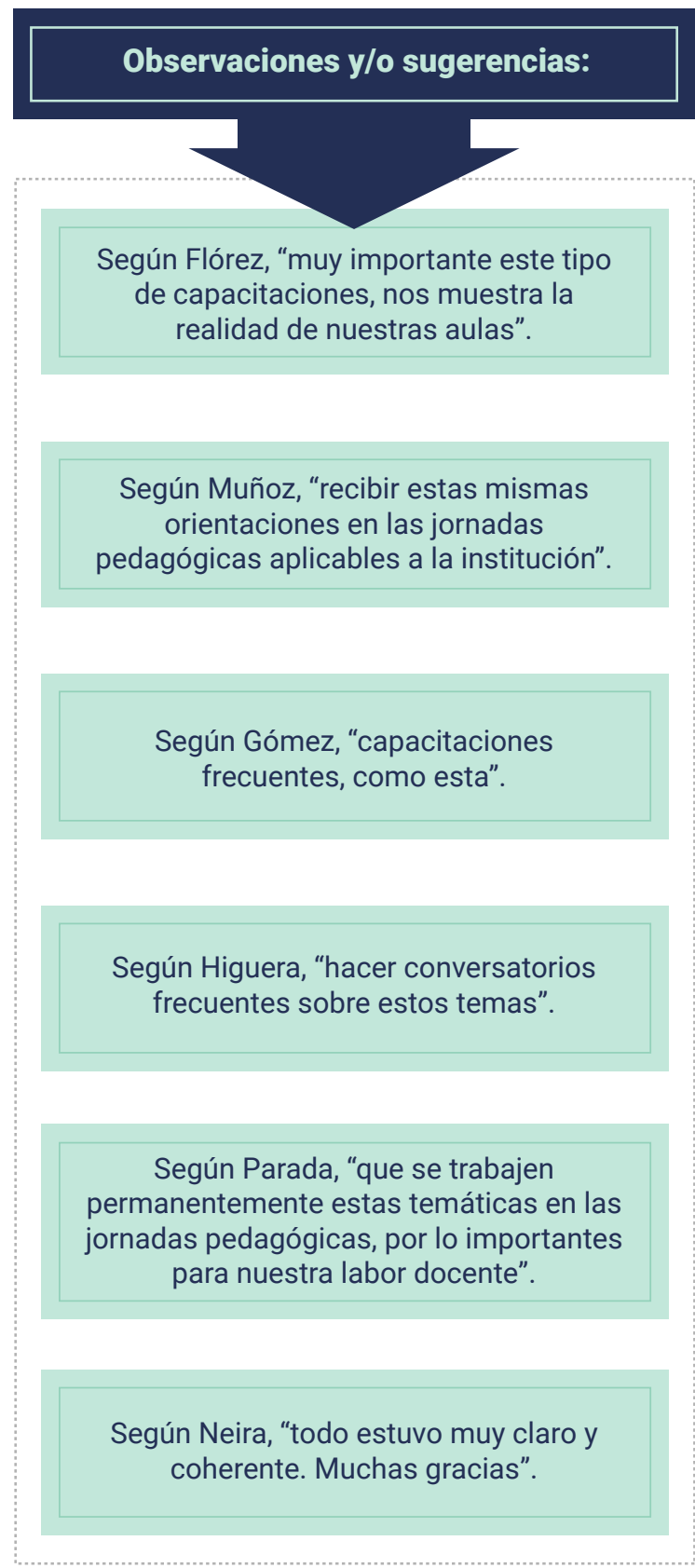

Sexto objetivo. Elaborar un "Plan de estrategias docentes para mejorar la motivación por el aprendizaje del inglés en los estudiantes de Media Vocacional $\left(10^{\circ}\right.$ y $\left.11^{\circ}\right)$ del Colegio Nuestra Señora de Fátima".
Como aporte se cumplió con la elaboración del "plan de estrategias docentes..." para ser puesto en práctica por los docentes a diario, en el ejercicio del acto didáctico y así mejorar la motivación de los estudiantes.

\section{Discusión}

Al relacionar los resultados cuantitativos se pueden reunir los hallazgos obtenidos, primero, en las preguntas de investigación aplicadas a los estudiantes: una motivación "regular", con un nivel de desmotivación "alto"; además, afirman que la desmotivación hacia el estudio del inglés depende del acto didáctico. Si tenemos en cuenta que la motivación según Maslow (1991, p. 264), se define como el impulso del ser humano para satisfacer sus necesidades.

\section{La motivación académica es el centro de energía, que impulsa a la acción, trabajo, estudio y a la realización plena del ser hu- mano. Equivale al motor que genera luz; si este no funciona, la luz eléctrica se desva- nece. De la misma manera ocurre con el estudio: si no hay motivación, el estudian- te no tendrá éxito. Cuanto más interés se tenga por el estudio, mejores resultados se obtendrán. (Borda \& Pinzón, 1995. p. 22)}

Según la RAE (2017), la desmotivación es "la falta o pérdida de la motivación”. Estamos ante una situación muy importante en la que se debe brindar atención y trabajar sin descanso para que los estudiantes salgan de este estado donde se encuentran.

La falta de motivación en el alumnado no solo supone un obstáculo para el aprendizaje de los conceptos si no también una dificultad para los docentes en su labor 
diaria de impartir clases, ya que los estudiantes pierden la atención y generan desorden con frecuencia. (Amores Valencia, 2020, párr. 2)

“En este ambiente de desmotivación las nuevas Tecnologías pueden desempeñar un papel fundamental, ya que se pueden considerar como una potente herramienta motivacional". (Hernández Sampieri, 2017, citado por Amores Valencia, 2020, párr. 3). Por tanto, la formación del profesorado en el uso de medios digitales y su uso en pos de la motivación son aspectos muy importantes en las nuevas metodologías en el aula.

A pesar de las discrepancias existentes, la mayoría de los especialistas coinciden en definir la motivación como "un conjunto de procesos implicados en la activación, dirección y persistencia de la conducta" (Beltrán, 1993a; Bueno, 1995; McClelland, 1989, etc., citado en García Bacete \& Doménech Betoret 1997, párr. 1).

Si nos centramos en el contexto escolar, el estudiante implicará en este proceso las actitudes, percepciones y expectativas que tenga en cuanto a la tarea, así mismo, a las metas que pretenda alcanzar, involucrando paralelamente el contexto en que se encuentre.

El aprendizaje y el grado en que los alumnos se interesan y se esfuerzan en aprender, es algo que se produce en un contexto. Los profesores al definir objetivos de aprendizaje, presentar información, proponer tareas, responder a las demandas de los alumnos, evaluar el aprendizaje de estos y ejercer el control y la autoridad, crean entornos de aprendizaje, que afectan no solo al aprendizaje, sino también a la motivación. (Tapia, 1995, p. 14)
Encontramos hallazgos por las preguntas de investigación aplicadas a los profesores: el rendimiento académico sobre todo en inglés es "bajo"; el nivel de calidad en que desarrollan el acto didáctico se ubica en "regular", y confirman que "la desmotivación por el aprendizaje del inglés depende del acto didáctico", todos los anteriores en el mayor porcentaje. En estos casos se recomienda el principio didáctico de fijación y retención del conocimiento; ya que sin una correcta fijación del conocimiento es imposible que el estudiante logre los hábitos y las habilidades que su trabajo futuro demanda de él y esto debe lograrse a toda costa si se utilizan las técnicas y los procedimientos a nuestra disposición en el aula.

En la teoría del aprendizaje significativo Ausubel (1983) plantea:

[...] que el aprendizaje del alumno depende de la estructura cognitiva previa que se relaciona con la nueva información, debe entenderse por "estructura cognitiva", al conjunto de conceptos, ideas que un individuo posee en un determinado campo del conocimiento, así como su organización. (p.1). Ausubel resume este hecho en el epígrafe de su obra de la siguiente manera: "Si tuviese que reducir toda la psicología educativa a un solo principio, enunciaría este: El factor más importante que influye en el aprendizaje es lo que el alumno ya sabe. Averígüese esto y enséñese consecuentemente". (p. 2)

Donn Byrne (1976), en su libro Teaching Oral English, nos dice que "es necesario buscar un equilibrio entre los niveles de corrección (dominio del sistema de la lengua) y la fluidez, de manera que los estudiantes puedan comunicarse adecuadamente, y que la inteligibilidad 
en el idioma va a estar marcada por el dominio que se tenga de la gramática, y el vocabulario". Por su parte Finnochiaro (1983) en su libro Functional-Notional Approach plantea que

el conocimiento "consciente o inconsciente" de los subsistemas de la lengua es necesario para hablar con fluidez y correctamente el idioma, comprender lo que escuchamos, leer con comprensión y escribir aceptablemente. Por tanto, es importante, entender que el uso de un enfoque comunicativo debe ir acompañado por cierto conocimiento del sistema de la lengua (no importa cómo) si tenemos en cuenta que los alumnos adultos aprenden más fácilmente por medio de enfoques cognitivos y académicos.

Seguidamente, en la aplicación de la escala de Likert, a los estudiantes, podemos responder a la pregunta: ¿de qué manera el acto didáctico es la causa de la desmotivación por el aprendizaje del inglés en los estudiantes del Nivel Media Vocacional $\left(10^{\circ}\right.$ y $11^{\circ}$ de Bachillerato) del Colegio Nuestra Señora de Fátima de Onzaga, Santander, Colombia? Se encuentra que los indicadores de los componentes del acto didáctico que más tienen que ver con la motivación o desmotivación del estudiante: el educador, recursos didácticos, estrategia didáctica, clima de aula, relación profesor alumno, reconocimiento y contexto familiar y del entorno, se ubican en el rango 1 (categorías muy baja, baja y media); mientras que los indicadores de los componentes: el discente, los objetivos de aprendizaje, el contexto y los contenidos, se ubican en el rango 2 (categorías Alta y Muy Alta). Lo anterior quiere decir que el acto didáctico es muy frío en su comunicación, pues esta no es la adecuada y, por tanto, hay una relación inversa y negativa entre los niveles de desmotivación de los estudiantes, y los niveles de calidad con que se desarrolla el acto didáctico: entre menos calidad del acto didáctico, mayor desmotivación de los estudiantes por el estudio del inglés.

Teniendo en cuenta la definición de acto didáctico de la Nación y de Contreras, ya mencionadas anteriormente en este documento, y los resultados encontrados, se concluye que el desarrollo del acto didáctico no cumple con la integridad con que se requiere como el estar revestido de atributos de calidad que lo hagan digno, formador, motivante y casi sagrado, y en el que se generen estrategias encaminadas a provocar el aprendizaje. Por esta razón se concluye que el acto didáctico es la causa de la desmotivación por el aprendizaje del inglés en los estudiantes, y es confirmado en un $100 \%$ por los profesores, luego de las sensibilizaciones.

Al desarrollar las sensibilizaciones, sobre autoevaluación del acto didáctico y profundización de los elementos clave del mismo, el profesorado participante demuestra inicialmente una actitud de rechazo, lo que no es desconocido para nadie debido a lo delicado del tema y a la alta sensibilidad del docente al cuestionarle su trabajo; sin embargo, la motivación es la clave para atraer la atención e ir facilitando la comunicación. Al evaluar estas temáticas se encuentran los siguientes hallazgos: el tema es claro, oportuno y muy importante. Se avanza en aceptar en un $100 \%$ la hipótesis presentada en la investigación: "la desmotivación hacia el aprendizaje del inglés depende del acto didáctico", y en un $100 \%$ los profesores manifiestan la necesidad de profundizar en los componentes clave del acto didáctico, petición atendida. Luego, al terminar, para el $100 \%$ del grupo participante los 
aportes son importantes, dejando, además, observaciones o sugerencias de valor para tener en cuenta.

Una vez conocida la situación de la investigación y para cumplir totalmente con el objetivo general, se elabora un "Plan de estrategias docentes para mejorar la motivación por el aprendizaje del inglés en los estudiantes de Media Vocacional $\left(10^{\circ}\right.$ y $\left.11^{\circ}\right)$ del Colegio Nuestra Señora de Fátima", para ser puesto en práctica a diario por los profesores, en el ejercicio del acto didáctico y así mejorar la motivación de los estudiantes y, desde luego, la del profesorado.

El plan de estrategias docentes contiene:

1. Estrategias docentes para conocer a los estudiantes. 2. Estrategias docentes para mantener el entusiasmo y dedicar tiempo a cada estudiante. 3. Estrategias docentes para mantener altas expectativas y señalar la importancia de la asignatura. 4. Estrategias docentes para mantener la confianza, la seguridad, la autoestima, el respeto y sobre todo la motivación del estudiante por el aprendizaje. En consecuencia, se puede concluir que:

1. Hay una alta incidencia del acto didáctico en la desmotivación por el estudio o aprendizaje del inglés.

2. Que la desmotivación por el estudio del inglés depende del acto didáctico (hipótesis comprobada).

3. La manera en que el acto didáctico es la causa de la desmotivación por el aprendizaje del inglés en los estudiantes del nivel Media Vocacional $\left(10^{\circ}\right.$ y $\left.11^{\circ}\right)$ del Colegio Nuestra Señora de Fátima, Onzaga, Santander, Colombia es porque este es muy frío humanamente, la comunicación no es adecuada, hay una relación inversa y negativa: entre menos calidad del acto didáctico, mayor desmotivación de los estudiantes por el estudio del inglés.

Todo lo anterior implica de los directivos un permanente acompañamiento a los profesores en el ejercicio de su misión docente para que el acto didáctico mejore en todas sus dimensiones, ya que han aceptado sus deficiencias, y quedan motivados para mejorar; también se necesita hacer gestión ante los diferentes entes: municipales, departamentales y nacionales para conseguir recursos que faciliten la capacitación y también la motivación para que se dé un acto didáctico de calidad, aprovechando el estado actual de motivación de los profesores. El impacto que se observará por el cambio beneficiará a toda la comunidad educativa del contexto y progresivamente a otras, municipales, departamentales y nacionales. Además, implica políticas de mayor atención a la educación en Colombia.

\section{Agradecimientos}

Agradezco, en primer lugar, a Dios que me ha permitido recorrer este camino y alcanzar esta meta tan deseada; a la Universidad Internacional Iberoamericana por abrirme las puertas y aceptarme como su estudiante; a mi directora de Tesis Dra. Juliana Azevedo Gomes, quien me ha compartido sus conocimientos para orientarme y llevar a feliz término mi tesis doctoral; a todo el personal directivo, docente y administrativo, de la Universidad, por sus calidades humanas; a mi familia, especialmente a mis hijos, Sandra Milena y Carlos Julián, por el apoyo incondicional en tiempo, comprensión y colaboración, para que yo pudiera dedicarme a este estudio; a mi esposo que desde el cielo 
me apoya, como lo hizo acá en la tierra; a la Institución Educativa Colegio Nuestra Señora de Fátima, que me permitió adelantar el trabajo de investigación; a los grupos participantes de profesores y estudiantes; y a todas las personas que en una o en otra forma aportaron a mi motivación para mantenerme en mi propósito. Mis sinceros agradecimientos a todos. Dios los bendiga.

\section{Referencias}

Amores Valencia, A. J. (2020). Las Nuevas Tecnologías como factor de motivación. Revista digital docente, (16). https://www. campuseducacion.com/blog/revista-digitaldocente/las-nuevas-tecnologias-como-factorde-motivacion/?cn-reloaded $=1$

Ausubel, D. (1983). Teoría del aprendizaje significativo. Fascículos de CEIF, 1(1-10), 1-10. 2).

Borda, E., \& Pinzón, B. (1995). La motivación y su relación con el aprendizaje del idioma inglés. Rendimiento Académico Técnicas para Estudiar Mejor. Cooperativa editorial magisterio.

Byrne, B. (1976), Enseñanza de inglés oral. Teaching Oral English. Longman. https:// openlibrary.org/books/OL7881780M/ Teaching_Oral_English.

Closer English, (Enero 22nd, 2019). Estudiar inglés potencia el cerebro de los estudiantes. https://www.closerenglish.com.co/ estudiar-ingles-potencia-cerebro/

Chaparro, L. (26 de abril de 2018). Las ventajas de un cerebro bilingüe. OpenMind. BBVA. Compartiendo conocimiento para un futuro mejor. Ciencia - Investigación, https://www. bbvaopenmind.com/ciencia/investigacion/ las-ventajas-de-un-cerebro-bilingue/
Contreras, D. 1994. Enseñanza, Curriculum y Profesorado. Introducción crítica a la didáctica (2. ${ }^{a}$ ed.). Universidad de Málaga. Departamento de didáctica y Organización Escolar.

Esparcia, A (2018, 13 de junio). La desmotivación escolar. Un tipo de fracaso. Revista Digital Docente. https://www.campuseducacion. com/blog/revista-digital-docente/ la-desmotivacion-escolar/

Finnocchiaro, M. (1983). Enfoque funcionalnocional: de la teoría a la práctica. FunctionalNotional Approach: From Theory to Practice.

\section{García Bacete, F. J., \& Doménech Betoret, F. D.} (1997). Motivación, aprendizaje y rendimiento escolar. Revista electrónica de motivación y emoción, 0(1). http://reme.uji.es/articulos/ pa0001/texto.html

Hernández Sampieri, R. (2017). Metodología de la investigación (6.a ed.). Mc Graw Hill Education.

Maslow, A. H, (1991). Motivación y personalidad (3. ${ }^{a}$ ed.). Ediciones Díaz de Santos, S.A.

La Nación. (Agosto 1 de 2017). El acto educativo de calidad. [Foros]. https://www.nacion.com/ opinion/foros/el-acto-educativo-de-calidad/4EU AUMNIHZAWDHBZAQT4QOJ3LI/story/

Otzen, T. \& Manterola C. Técnicas de muestreo sobre una población a estudio. Int. J. Morphol., 35(1), 227-232, 2017. http://dx.doi.org/10.4067/ S0717-95022017000100037

RAE, 2017. Desmotivación. Real Academia Española. Madrid, España.https://dle.rae.es/ desmotivaci\%C3\%B3n

Sabino, C. (1996). El proceso de investigación. Editorial Panapo.http://paginas.ufm.edu/sabino/ ingles/book/proceso_investigacion.pdf

Smart Academia. (2021). Cómo reacciona tu CEREBRO cuando aprendes IDIOMAS [Video]. 17 abril 2021. https://www.youtube.com/ watch?v=2m1ecwvlQ7c 
Tapia, A. (1995). La motivación o desmotivación se produce en interacción con el contexto. Motivar para el aprendizaje. Teoría y Estrategias. http://www.terras.edu.ar/biblioteca/6/TA_Tapia_ Unidad_4.pdf

Vargas Soto. A. (2017). 10 razones para aprender el idioma inglés. Boletín Científico Semestral de la Universidad Autónoma del Estado de Hidalgo, 4(8). https://www.uaeh.edu.mx/scige/boletin/ prepa2/n8/p2.html\#r1
Vega B, F, 2020. ¿Qué pasa en tu cerebro cuando aprendes otro idioma? Mitos y realidades.

UNAM Global. Universidad Nacional Autónoma de México. https://www.youtube.com/ watch?v=BrUc7rNonWM 